\title{
Unlocking Synergy in Bimetallic Catalysts by Core-Shell Design
}

\author{
Jessi E.S. van der Hoeven ${ }^{1,2}$, Jelena Jelic ${ }^{3}$, Liselotte A. Olthof ${ }^{1,2}$, Giorgio Totarella ${ }^{1}$, Relinde \\ J.A. van Dijk-Moes ${ }^{2}$, Felix Studt ${ }^{3,4}$, Alfons van Blaaderen ${ }^{2}$, and Petra E. de Jongh ${ }^{{ }^{*}}$ \\ ${ }^{1}$ Inorganic Chemistry and Catalysis, Debye Institute for Nanomaterials Science, Utrecht \\ University, Universiteitsweg 99, 3584 CG Utrecht, The Netherlands \\ ${ }^{2}$ Soft Condensed Matter, Debye Institute for Nanomaterials Science, Utrecht University, \\ Princetonplein 5, 3584 CC Utrecht, The Netherlands \\ ${ }^{3}$ Institute of Catalysis Research and Technology, Karlsruhe Institute of Technology, \\ Herrmann-von-Helmholtz-Platz 1, 76344 Eggenstein-Leopoldshafen, Germany \\ ${ }^{4}$ Institute for Chemical Technology and Polymer Chemistry, Karlsruhe Institute of \\ Technology (KIT), Engesserstraße 18-20, 76131 Karlsruhe, Germany \\ ${ }^{*}$ E-mail: A.vanBlaaderen@uu.nl, P.E.deJongh@uu.nl
}

\begin{abstract}
Extending the toolbox from mono- to bimetallic catalysts is key in realizing efficient chemical processes [1]. Traditionally, the performance of bimetallic catalysts featuring one active and one selective metal is optimized by varying the metal composition $[1,2,3]$, often resulting in a compromise between the catalytic properties of the two metals $[4,5,6]$. Here we show that by designing the atomic distribution of bimetallic Au-Pd nanocatalysts, we obtain a synergistic catalytic performance in the industrially relevant selective hydrogenation of butadiene. Our single crystalline Au-core Pdshell nanorods were up to 50 times more active than their alloyed and monometallic counterparts, while retaining high selectivity. We find a shell thickness dependent catalytic activity, indicating that not only the nature of the surface but also several sub-surface layers play a crucial role in the catalytic performance, and rationalize this finding using density-functional-theory calculations. Our results open up a novel avenue for the structural design of bimetallic catalysts.
\end{abstract}

Synergy arises when two catalytically active metals are combined such that the catalytic performance exceeds that of the monometallic counterparts [1]. This makes bimetallics an interesting class of materials for catalyzing a variety of chemical processes ranging from selective hydrogenation $[1,5,7]$, to oxidation $[2,4,8]$ and electrochemical reactions $[9,10,11]$. The main focus has been on alloyed nanoparticles, as they are easily accessible with standard catalyst preparation methods and allow facile tuning of their catalytic properties via the average metal composition. However, also the arrangement in which the atoms are assembled is crucial; gas- and thermally-induced metal redistribution can have a large impact on the catalytic performance $[12,13,14]$. Thanks to recent advances in ma- terial science, it is now possible to synthesize bimetallic nanoparticles with precisely defined atomic arrangements such as single-atom alloys [15, 16], intermetallic structures [17] and core-shell materials [18, 19]. Yet, the number of systematic studies linking the metal distribution to the performance of bimetallic catalysts is limited $[12,13,14]$. In particular, the catalytic behavior of core-shell nanoparticles is largely unexplored, despite successful demonstrations of core-shell catalysts in electrocatalysis, where changes in the electronic properties of the shell atoms induced by the underlying core resulted in enhanced catalytic performances $[9,10,11,20]$.

Here, by employing colloid synthesis $[19,21]$, we prepared a well-defined Au-Pd model system with a precisely tunable atomic structure allowing a direct correlation between the metal distribution, composition, crystal structure, and the catalytic performance. Specifically, we test these catalysts in the selective hydrogenation of 1,3-butadiene, which is a crucial reaction in purifying alkene feedstock for polymer industry. The challenge is to selectively convert polyolefins, without hydrogenation of mono-olefins which are in large excess. Pd-based materials are active hydrogenation catalysts as hydrogen is easily dissociated on the Pd-surface [22]. At low conversions, Pd catalysts can be quite selective [23]. However, near full conversion the hydrogenation of mono-olefins becomes increasingly important [24]. By combining $\mathrm{Pd}$ with a less active, more selective metal like $\mathrm{Au}$, or poisoning it with sulfur such over-hydrogenation can be suppressed [1, 25, 26, 27, 28], but not without compromising activity compared to pure $\mathrm{Pd}[5,29]$.

In this work, we designed model catalysts consisting of Au-core Pd-shell nanorods coated with a protective mesoporous silica shell (Au@Pd@ $\mathrm{SiO}_{2} \mathrm{NRs}$ ) as schematically shown in Figure 1a. The AuNRs have a single crystalline FCC structure with $\{110\}$ and $\{100\}$ surface facets exposed along the length of the rod [30]. 
The Pd-shell thickness was varied by changing the Pdprecursor $\left(\mathrm{NaPdCl}_{4}=0-0.08 \mathrm{mM}\right)$ to obtain NRs with different shell thicknesses (Figure 1b). This resulted in $\mathrm{Au} @ \mathrm{Pd} @ \mathrm{SiO}_{2} \mathrm{NRs}$ with an atomic Pd fraction $X_{P d}=$ $0.04,0.08,0.21$ and 0.32 corresponding to $N_{P d}=1,2,5$ and 6 Pd layers. We ensured that the different batches of $\mathrm{Au} @ \mathrm{Pd} @ \mathrm{SiO}_{2}$ had a similar volume by performing the metal overgrowth for the low Pd content samples directly on the mesoporous silica coated AuNRs and for the highest Pd content NRs on smaller, etched $\mathrm{Au}-$ cores.

The high-angle annular dark-field scanning transmission electron microscopy (HAADF-STEM) images, reported in Figure 1c-e confirm that the NRs were monodisperse in size and shape, and were $\sim 70 \mathrm{~nm}$ and $20 \mathrm{~nm}$ in length and diameter, respectively (see Supplementary Table S2). The energy-dispersive X-ray spectroscopy (EDX) maps, depicting a 2D projection of the elemental distribution $(\mathrm{Au}, \mathrm{Pd}$, and $\mathrm{Si}$, are shown in red, green and blue, respectively), clearly reveal the $\mathrm{Au}$-core Pd-shell structure and show that the rods were homogeneous in composition meaning that every particle had a similar Pd-content in contrast to most catalysts reported in literature and prepared by more conventional methods. Note that there is a slightly higher Pd content at the NR tips, which can be homogenized via mild thermal treatment at $250{ }^{\circ} \mathrm{C}$ in $\mathrm{H}_{2}$, resulting in a smooth, epitaxial $\mathrm{Pd}$ on $\mathrm{Au}$ shell (Supplementary Figure 1). The Si signal in the EDX maps shows that each NR was encapsulated with a $18 \mathrm{~nm}$ thick mesoporous silica shell. This shell protected the particles from deformation and sintering in the subsequent catalytic testing, whereas the mesopores ensured mass transport to the metal surface. To render $\mathrm{Au} @ \mathrm{Pd} @ \mathrm{SiO}_{2}$ NRs suitable for gas phase catalysis, the organic template and ligand molecules were removed from the mesopores by washing with acidified ethanol (Supplementary Figure 2) followed by deposition of the NRs on a commercial silica support (Supplementary Figure 3). The final metal loading of the catalysts was $0.02 \mathrm{wt} \%$.

We tested the catalyst performance of the $\mathrm{Au} @ \mathrm{Pd} @ \mathrm{SiO}_{2}$ NRs in the selective hydrogenation of butadiene to butene in the presence of an excess of propene. While keeping the metal composition of the NRs fixed at $X_{P d}=0.08\left(N_{P d}=2\right)$, we studied the effect of the metal distribution on the catalyst performance. Upon increasing temperature, the metal structure of the bimetallic NRs changed from coreshell to fully alloyed, as evident from the EDX maps reported in Figure 2a, with intermediate partially alloyed structures such as the one at $350{ }^{\circ} \mathrm{C}$ (see Supplementary Figure 4-5 for quantification of the alloying process). Notably, the NRs exhibited an exceptionally high thermal stability, enabling full retention of the particle shape while changing the atomic distribution within the nanoparticles. In Figure $2 \mathrm{~b}$, we show the catalytic activity of the differently pretreated $\mathrm{Au} @ \mathrm{Pd} @ \mathrm{SiO}_{2}$ NRs at a reaction temperature of 60 ${ }^{\circ} \mathrm{C}$. The catalytic activity is given as a turnover frequency (TOF) which is expressed as the number of butadiene molecules converted per second per metal surface atom. The TOFs of the core-shell structured $\mathrm{Au} @ \mathrm{Pd} @ \mathrm{SiO}_{2} \mathrm{NRs}\left(T_{p r e}=250-300{ }^{\circ} \mathrm{C}\right)$ were up to 32 times higher than the partially and fully alloyed $\left(T_{p r e}=\right.$ 350-450 ${ }^{\circ} \mathrm{C}$ ) AuPd@ $\mathrm{SiO}_{2}$ NRs, which can be explained by the diffusion of less active Au atoms to the NR surface. Furthermore, the butadiene conversion versus temperature curves (Figure 2c) show that the coreshell structured NRs were already active at room temperature, even though the metal loading was as low as $0.02 \mathrm{wt} \%$ and the particle size relatively large. In addition to the excellent activity, the core-shell NRs exhibited a remarkably high selectivity (Figure $2 \mathrm{~b}$ and Supplementary Figure 6) and highly reproducible catalytic performance (Supplementary Figure 7). Herein, the selectivity was defined as the number of hydrogen molecules used for the conversion of butadiene to butene divided by the total number of consumed hydrogen molecules. In Figure 2b, we deliberately show the selectivity at high butadiene conversion (98\%) as maintaining high selectivity at low butadiene concentrations is most challenging. Figure $2 \mathrm{~d}$ displays the selectivity of the differently structured $\mathrm{Au} @ \mathrm{Pd} @ \mathrm{SiO}_{2}$ NRs over the full range of butadiene conversions, showing that the selectivity was larger than $75 \%$ for all samples and highest for the alloyed, followed by the partially alloyed and then the core-shell catalysts.

As the core-shell structure combined an extraordinary high activity while retaining high selectivity, we further investigated the core-shell structures and studied the influence of the shell thickness on the catalytic behavior. Herein, NRs with 1, 2, 5, $6 \mathrm{Pd}$ shell layers and $X_{P d}=0.04,0.08,0.21,0.32$ were used and compared to a $\mathrm{Au}$ and $\mathrm{Pd}$ reference catalyst. As it was not feasible to synthesize well-defined monometallic Pd rods, we prepared a catalyst consisting of spherical Pd NPs with an average diameter of $6.1 \mathrm{~nm}$ on a silica support as a reference (Supplementary Figure 8). Furthermore, the monometallic Au nanorods were not active enough (conversion $\leq 20 \%$ ) to compare them to the $\mathrm{Au}-\mathrm{Pd}$ and Pd catalyst at the same temperature interval (Supplementary Figure 9). We therefore included the catalytic performance of a $\mathrm{Au}$ on silica catalyst $(\mathrm{d}=$ $3.2 \mathrm{~nm}, 3.6 \mathrm{wt} \%$ ) from literature in Figure 3 for comparison [28]. In Figure 3 we show that the bimetallic core-shell NRs outperformed the monometallic Au and Pd catalysts and that the catalytic performance was highly sensitive to the Pd-shell thickness. In Figure 3a the catalytic activity, expressed as TOF, at a reaction temperature of $45{ }^{\circ} \mathrm{C}$ and the selectivity at high (98\%) butadiene conversion are plotted as a function of the atomic Pd fraction $\left(X_{P d}\right)$. All core-shell structures with more than $1 \mathrm{Pd}$ layer were considerably more active than the monometallic Au and Pd NPs and exhibited very high TOFs. For comparison, the TOF of much smaller, 2-3 nm Au-Pd nanoparticles on silica $\left(X_{P d}=0.05\right)$ tested under the same conditions was 0.001 to $0.1 \mathrm{~s}^{-1}$ at $60{ }^{\circ} \mathrm{C}$ [29]. The high activity of the $\mathrm{Au} @ \mathrm{Pd} @ \mathrm{SiO}_{2}$ catalysts is confirmed by the conversion profiles in Figure 3b. Interestingly, the activity of the Au@Pd@SiO ${ }_{2}$ NRs depended non-monotonically 
on the shell thickness, with an optimal activity at 6 atomic Pd layers. The selectivity was highest for the pure $\mathrm{Au}$ and $\mathrm{Au} @ \mathrm{Pd} @ \mathrm{SiO}_{2} \mathrm{NRs}$ with $N_{P d}=1$ and lowest for the pure Pd sample (Figure $3 c$ and Supplementary Figure 10). By plotting the selectivity as a function of conversion we show that the selectivity drops to lower values for all catalyst at high conversion, yet remained above $80 \%$ for the $\mathrm{Au} @ \mathrm{Pd} @ \mathrm{SiO}_{2} \mathrm{NRs}$ with $N_{P d}=1$ and 6 . This means that the best combined activity and selectivity was obtained with the core-shell catalyst with $N_{P d}=6$, where the TOF was $50 \mathrm{~s}^{-1}$ at $45{ }^{\circ} \mathrm{C}$ and the selectivity $80 \%$ at $98 \%$ butadiene conversion.

To understand the catalytic behavior of the coreshell NRs, we employed density-functional-theory calculations (DFT) and studied the effect of the surface facets, Pd-shell thickness and lattice strain on the adsorption energies of butadiene, propene, butene and hydrogen. From our reaction order measurements (Supplementary Figure 11), it follows that the catalytic activity depends on the butadiene and hydrogen pressure, and is insensitive to the propene pressure. We therefore studied the adsorption energy of butadiene (Figure 4a) and hydrogen (Supplementary Figure 12) as a function of the Pd-shell thickness for face centered cubic (FCC) $\{100\},\{111\}$ and $\{110\}$ terminated surfaces. For butadiene, we find a strong dependence on the type of surface facet, with the $\{110\}$ surface binding butadiene the strongest. Our results are consistent with previous studies $[24,31,32]$ on $\mathrm{Pd}$ and $\mathrm{Au}-\mathrm{Pd}$ single crystals reporting higher reaction rates at the $\{110\}$ surface. The $\{110\}$ surface is therefore likely to dominate the catalytic behavior of the Au@Pd@ $\mathrm{SiO}_{2}$ NRs. Furthermore, since a large part of the NR surface is composed of $\{110\}$ facets and the activity of $\mathrm{Pd}$ catalysts is mostly particle size independent [24], the high adsorption energies at the $\{110\}$ facets could, in part, explain the high catalytic activity of our coreshell NRs.

Next, we studied the effect of the shell-thickness on the adsorption energies of all reactants on a $\{110\}$ terminated surface (Figure 4b, see Supplementary Figure 13 for the $\{111\}$ and $\{100\}$ surfaces). The $\sim 1 \mathrm{eV}$ difference in binding energy between butadiene and propene/butene explains the high selectivity of $\mathrm{Au} @ \mathrm{Pd} @ \mathrm{SiO}_{2}$ NRs as observed experimentally. The increased binding strength of butadiene and hydrogen on the layered $\mathrm{Au}-\mathrm{Pd}$ structures compared to pure $\mathrm{Au}$ explains the increased activity of the core-shell catalysts. The high adsorption energies of the reactants on the core-shell compared to the monometallic catalysts are likely caused by lattice strain stemming from the lattice mismatch between the Au core and Pd overlayer $(\sim 4 \%)$. This is supported by the DFT results in Figure $4 \mathrm{c}$ showing the hydrogen and butadiene adsorption energy for pure $\mathrm{Pd}$ with a Pd lattice constant and for strained Pd with an Au lattice constant. Lattice strain induced changes in reactant binding energies have been observed before, for instance in the oxygen reduction reaction on strained core-shell catalysts $[9,11]$. Finally, we note that the shell thickness dependent catalytic ac- tivity as measured experimentally (Figure 3) does not directly correspond to the present DFT calculations. The $\mathrm{Au} @ \mathrm{Pd} @ \mathrm{SiO}_{2}$ NRs with $N_{P d}=1$ and 2 are less active than might be expected based on the hydrogen and butadiene adsorption energies. Although the EDX analysis revealed an overall core-shell structure after pretreatment at $250{ }^{\circ} \mathrm{C}$ (Supplementary Figure 4), we cannot exclude a slight redistribution at the nanorod surface for the Au@Pd@ $\mathrm{SiO}_{2}$ NRs with $N_{P d}=1$ and 2 , rendering a less pure $\mathrm{Pd}$-shell and hence a less active catalyst compared to the $\mathrm{Au} @ \mathrm{Pd} @ \mathrm{SiO}_{2} \mathrm{NRs}_{\text {with }}$ $N_{P d}=5$ and 6 .

Altogether, our results show three key features: i) the core-shell catalysts are considerably more active than their alloyed counterparts, ii) the activity and selectivity of the core-shell catalysts are shell thickness dependent and iii) the properties of Au-core Pd-shell catalysts do not resemble those of pure Pd even when the Pd-shell is as thick as 6 atomic layers. We ascribe these observations to i) strong binding of the reactants to the $\{110\}$ facets running along the sides of the AuNRs and ii) strain induced changes in the butadiene and hydrogen adsorption energies on Au-core Pd-shell nanostructures. Our study highlights the importance of tuning the atomic distribution in bimetallic catalysts, and lays a foundation for the rational design of bimetallic catalysts with optimal synergistic performances. 

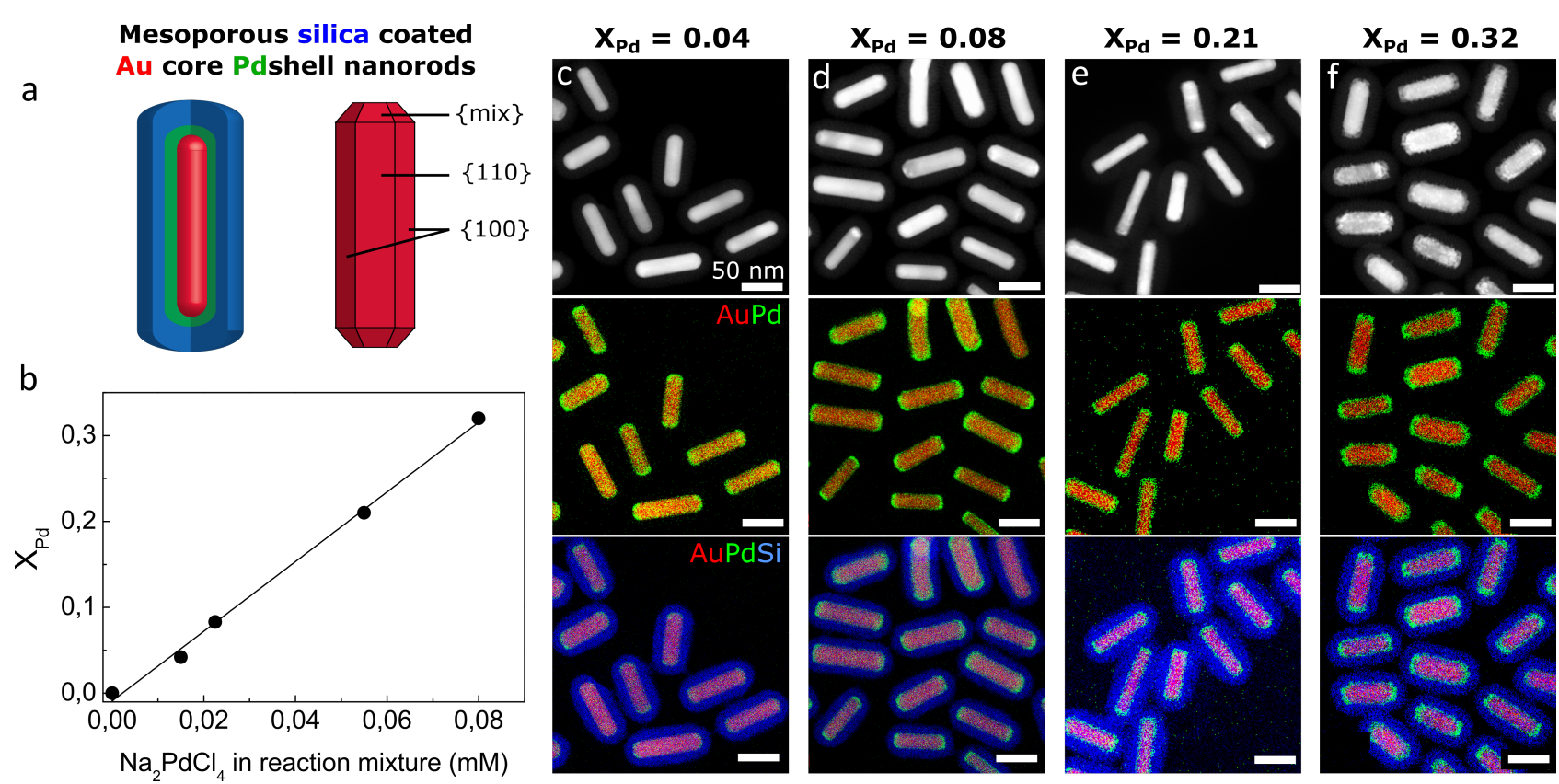

Figure 1: Our model system of monodisperse Au@Pd@SiO ${ }_{2}$ NRs with controlled Pd content and shell thickness. a) Schematic representation of the $\mathrm{Au} @ \mathrm{Pd} @ \mathrm{SiO}_{2} \mathrm{NRs}$ and their surface structure. b) Atomic $\mathrm{Pd}$ fraction as a function the $\mathrm{Pd}$-precursor $\left(\mathrm{Na}_{2} \mathrm{PdCl}_{4}\right)$ concentration present in the synthesis mixture. c-e) HAADF-STEM and EDX maps showing the structure and composition of Au@Pd@SiO $\mathrm{NRs}$ with $X_{P d}=0.02$ (c), 0.04 (d), 0.21 (e) and 0.32 (f). In the EDX maps Au, Pd and Si are presented in red, green and blue, respectively.
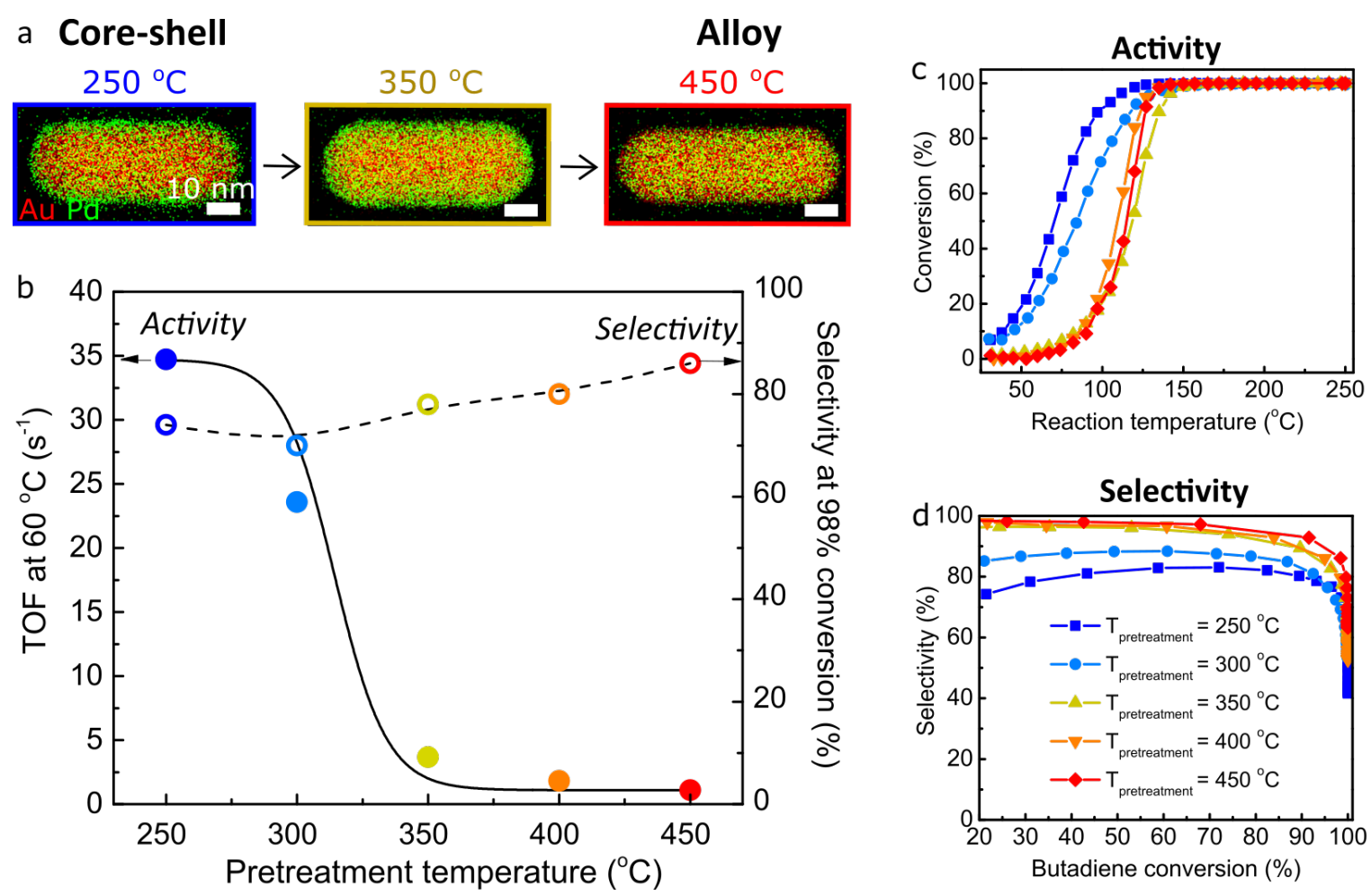

Figure 2: Core-shell structured Au-Pd catalysts outperform their alloyed counterparts. a) EDX maps showing core-shell $\left(250{ }^{\circ} \mathrm{C}\right)$, partially alloyed $\left(350{ }^{\circ} \mathrm{C}\right)$ and alloyed $\left(450{ }^{\circ} \mathrm{C}\right) \mathrm{AuPd} @ \mathrm{SiO}_{2}$ NRs with $X_{P d}=$ 0.08 and $N_{P d}=2$. b) Catalytic activity and selectivity of the differently structured $\mathrm{AuPd} @ \mathrm{SiO}_{2} \mathrm{NRs}$ in the selective hydrogenation of butadiene. c) Butadiene conversion as a function of reaction temperature of the $\mathrm{AuPd} @ \mathrm{SiO}_{2} \mathrm{NRs}$ pretreated at different temperatures. c) Selectivity as a function of the butadiene conversion. For all catalytic tests $20 \mathrm{mg}$ catalysts with $0.02 \mathrm{wt} \%$ metal was used). The reaction mixture consisted of $0.3 \%$

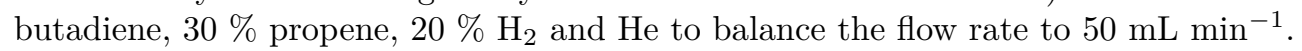



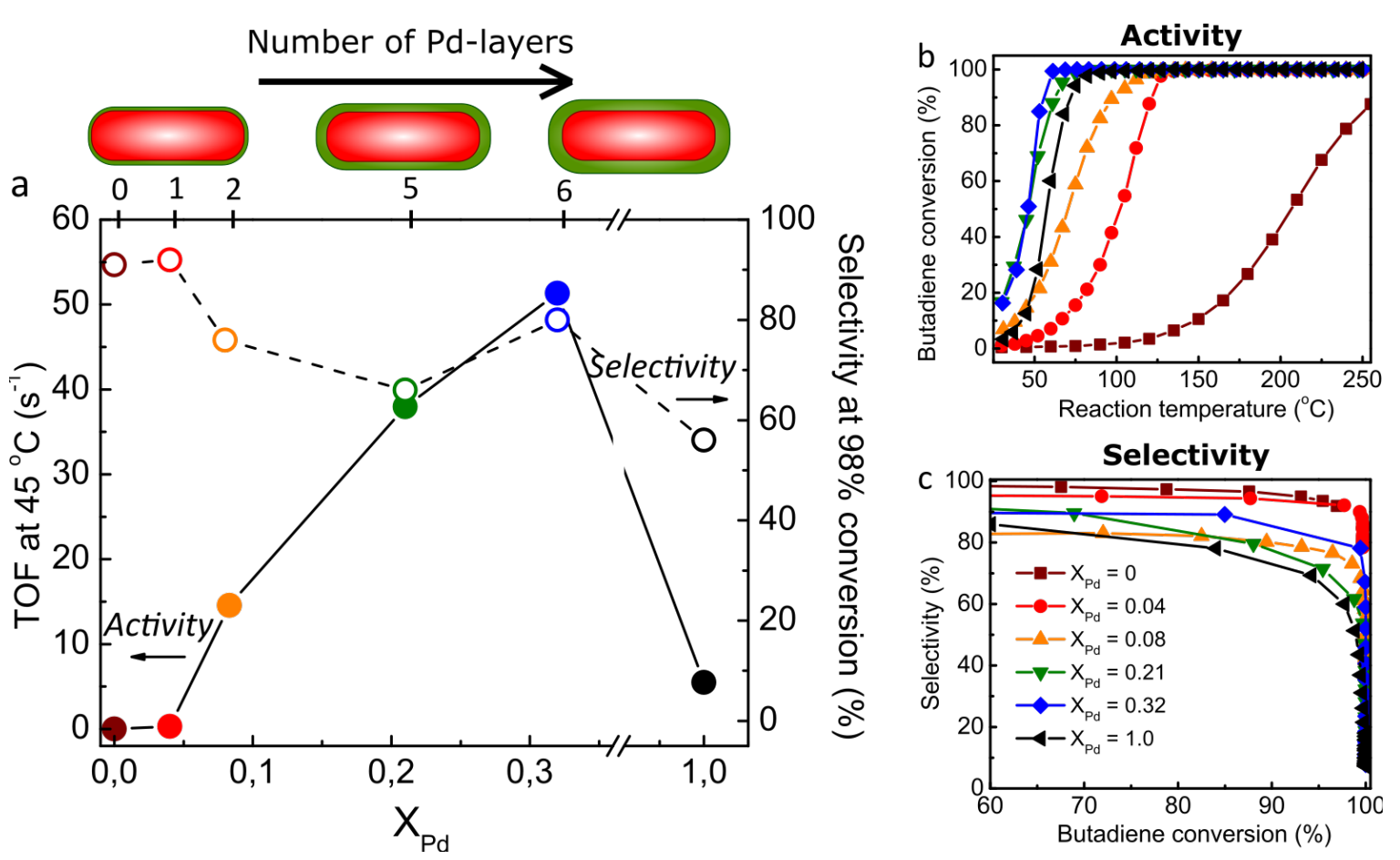

Figure 3: The catalytic performance of Au-core Pd-shell catalysts is highly sensitive to the number of shell layers. $\mathrm{Pd} @ \mathrm{SiO}_{2}$ NRs with variable Pd-content and shell thickness were used: $X_{P d}=0.04$ $/ N_{P d}=1$ (red), $X_{P d}=0.08 / N_{P d}=2$ (orange), $X_{P d}=0.21 / N_{P d}=5$ (green), $X_{P d}=0.32 / N_{P d}=6$ (blue), and a $\mathrm{Au} @ \mathrm{SiO} 2\left(X_{A u}=1.0\right.$, brown $)$ and $\mathrm{Pd} @ \mathrm{SiO}_{2}\left(X_{P d}=1.0\right.$, black $)$ reference sample containing spherical 3.0 and 6.1 $\mathrm{nm}$ particles, respectively. a) Activity expressed as TOF $\left(\mathrm{s}^{-1}\right)$ at $45{ }^{\circ} \mathrm{C}$ (left axis) and the selectivity at $98 \%$ butadiene conversion (right axis) as a function of the atomic Pd-fraction. b) Butadiene conversion as a function of the reaction temperature. c) Selectivity as a function of the butadiene conversion. The metal loading for the $\mathrm{Au} @ \mathrm{Pd}$ NRs with $X_{P d}=0.04-0.32$ and $X_{P d}=0.02$ was 0.02 wt\% and $0.2 \mathrm{wt} \%$, respectively. The metal loading for the $\mathrm{Au} @ \mathrm{SiO}_{2}$ and $\mathrm{Pd} @ \mathrm{SiO}_{2}$ reference samples was 3.6 and $0.0065 \mathrm{wt} \%$.
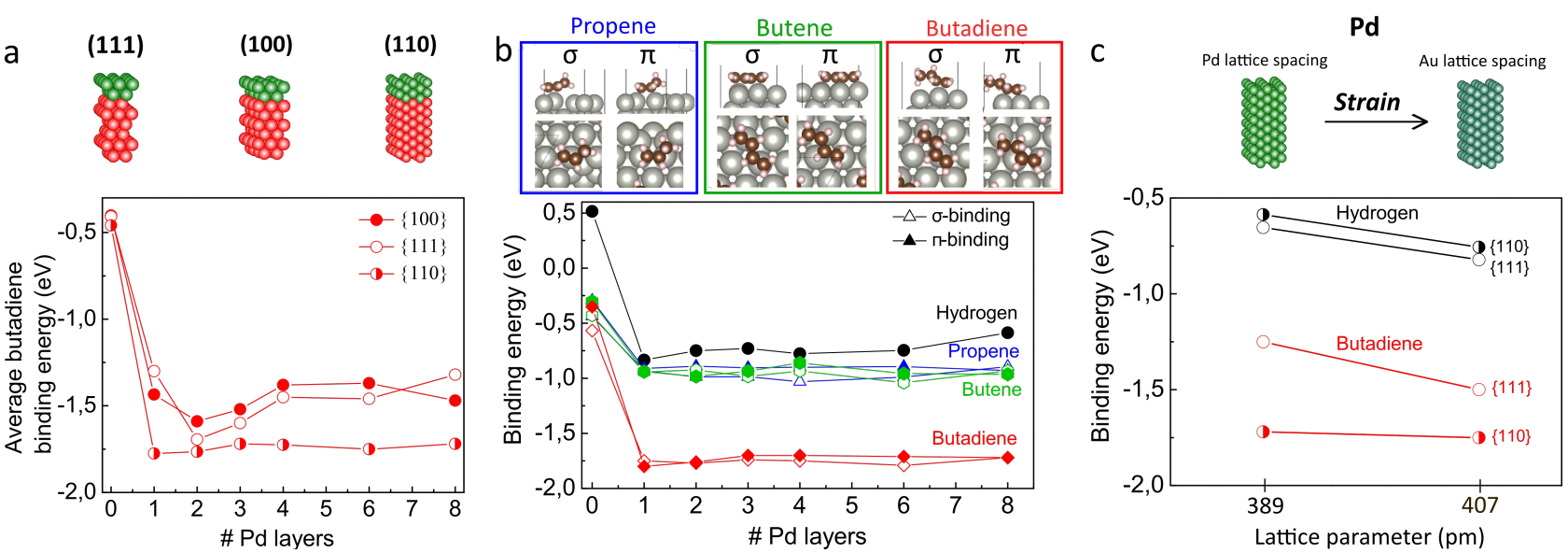

Figure 4: Crystallographic orientation of the surface facets, Pd-shell thickness and lattice strain govern the reactant adsorption energies. a) Average adsorption energy of butadiene as a function of the number of Pd-layers at a $\{100\},\{111\}$ and $\{110\}$ surface. b) Schematic representation of the $\sigma$ and $\pi$ binding modes and plot of the binding energy as a function of the Pd-shell thickness of hydrogen (black), propene (blue), butene (green) and butadiene (red). c) Hydrogen and butadiene adsorption on Pd and strained Pd $\{111\}$ and $\{110\}$ surfaces with a $\mathrm{Pd}(389 \mathrm{pm})$ and Au lattice parameter $(407 \mathrm{pm})$, respectively. 


\section{References}

[1] Zhang, L., Zhou, M., Wang, A. \& Zhang, T. Selective hydrogenation over supported metal catalysts: from nanoparticles to single atoms. Chemical Reviews (2019).

[2] $\mathrm{Wu}, \mathrm{C} . \mathrm{H}$. et al. Bimetallic synergy in cobaltpalladium nanocatalysts for CO oxidation. Nature Catalysis 2, 78-85 (2019).

[3] Studt, F. et al. Identification of non-precious metal alloy catalysts for selective hydrogenation of acetylene. Science 320, 1320-1322 (2008).

[4] Enache, D. I. et al. Solvent-free oxidation of primary alcohols to aldehydes using $\mathrm{Au}-\mathrm{Pd} / \mathrm{TiO}_{2}$ catalysts. Science 311, 362-365 (2006).

[5] Hugon, A., Delannoy, L., Krafft, J.-M. \& Louis, C. Selective hydrogenation of 1,3-butadiene in the presence of an excess of alkenes over supported bimetallic gold palladium catalysts. The Journal of Physical Chemistry C 114, 10823-10835 (2010).

[6] Masoud, N., Delannoy, L., Calers, C. \& Gallet, J. J. Silica-Supported Au-Ag Catalysts for the Selective Hydrogenation of Butadiene. Chem. Cat. Chem. 9, 2418-2425 (2017).

[7] Lucci, F. R. et al. Selective hydrogenation of 1,3butadiene on platinum-copper alloys at the singleatom limit. Nature Communications 6, 8550 (2015).

[8] Wang, G.-H. et al. Platinum-cobalt bimetallic nanoparticles in hollow carbon nanospheres for hydrogenolysis of 5-hydroxymethylfurfural. Nature Materials 13, 293-300 (2014).

[9] $\mathrm{Bu}$, L. et al. Biaxially strained $\mathrm{PtPb} / \mathrm{Pt}$ core/shell nanoplate boosts oxygen reduction catalysis. Science 354, 1410-1414 (2016).

[10] Wang, X. et al. Palladium-platinum core-shell icosahedra with substantially enhanced activity and durability towards oxygen reduction. Nature Communications 6, 7594 (2015).

[11] Strasser, P. et al. Lattice-strain control of the activity in dealloyed core-shell fuel cell catalysts. $\mathrm{Na}$ ture Chemistry 2, 454-460 (2010).

[12] Zugic, B. et al. Dynamic restructuring drives catalytic activity on nanoporous gold-silver alloy catalysts. Nat. Mater. 16, 558-565 (2016).

[13] Destro, P. et al. The crucial role of the support in the transformations of bimetallic nanoparticles and catalytic performance. ACS Catal. 8, 10311037 (2018).
[14] Ahmadi, M., Behafarid, F., Cui, C., Strasser, P. \& Cuenya, B. R. Long-Range Segregation Phenomena in Shape-Selected Bimetallic Nanoparticles: Chemical State Effects. ACS Nano 7, 91959204 (2013).

[15] Wrasman, C. J. et al. Synthesis of colloidal pd/au dilute alloy nanocrystals and their potential for selective catalytic oxidations. Journal of the American Chemical Society 140, 12930-12939 (2018).

[16] Luneau, M. et al. Dilute $\mathrm{Pd} / \mathrm{Au}$ alloy nanoparticles embedded in colloid-templated porous $\mathrm{SiO}_{2}$ : Stable Au-based oxidation catalysts. Chemistry of Materials 31, 5759-5768 (2019).

[17] Lee, J. D. et al. Tuning the electrocatalytic oxygen reduction reaction activity of $\mathrm{Pt}-\mathrm{Co}$ nanocrystals by cobalt concentration with atomic-scale understanding. ACS Applied Materials \& Interfaces 11, 26789-26797 (2019).

[18] Gilroy, K. D., Ruditskiy, A., Peng, H.-C., Qin, D. \& Xia, Y. Bimetallic nanocrystals: Syntheses, properties, and applications. Chemical Reviews 116, 10414-10472 (2016).

[19] Deng, T. S. et al. Oxidative etching and metal overgrowth of gold nanorods within mesoporous silica shells. Chem. Mater. 27, 7196-7230 (2015).

[20] Zhang, Y.-J. et al. Probing the electronic structure of heterogeneous metal interfaces by transition metal shelled gold nanoparticle-enhanced raman spectroscopy. The Journal of Physical Chemistry C 120, 20684-20691 (2016).

[21] van der Hoeven, J. E. S. et al. In situ observation of atomic redistribution in alloying gold-silver nanorods. ACS Nano 12, 8467-8476 (2018).

[22] Yu, W.-Y., Mullen, G. M. \& Mullins, C. B. Hydrogen adsorption and absorption with $\mathrm{Pd}-$ Au bimetallic surfaces. The Journal of Physical Chemistry C 117, 19535-19543 (2013).

[23] Molnár, A., Sárkány, A. \& Varga, M. Hydrogenation of carbon-carbon multiple bonds: chemo-, regio- and stereo-selectivity. Journal of Molecular Catalysis A: Chemical 173, 185 - 221 (2001).

[24] Silvestre-Albero, J., Rupprechter, G. \& Freund, H.-J. From pd nanoparticles to single crystals: 1,3-butadiene hydrogenation on well-defined model catalysts. Chem. Commun. 80-82 (2006).

[25] Bachiller-Baeza, B. et al. Detecting the genesis of a high-performance carbon-supported Pd sulfide nanophase and its evolution in the hydrogenation of butadiene. ACS Catalysis 5, 5235-5241 (2015).

[26] McEwan, L., Juliusa, M., Robertsa, S. \& Fletchera, J. C. Q. A review of the use of gold catalysts in selective hydrogenation reactions. Gold Bulletin 43, 298-306 (2010). 
[27] Hugon, A., Delannoy, L. \& Louis, C. Supported gold catalysts for selective hydrogenation of 1,3butadiene in the presence of an excess of alkenes. Gold Bulletin 41, 127-138 (2008).

[28] Masoud, N. et al. Superior stability of $\mathrm{Au} / \mathrm{SiO}_{2}$ compared to $\mathrm{Au} / \mathrm{TiO}_{2}$ catalysts for the selective hydrogenation of butadiene. ACS Catalysis 7, 5594-5603 (2017).

[29] Kolli, N. E., Delannoy, L. \& Louis, C. Bimetallic $\mathrm{Au}-\mathrm{Pd}$ catalysts for selective hydrogenation of butadiene: Influence of the preparation method on catalytic properties. Journal of Catalysis 297, 79 - 92 (2013).

[30] Goris, B. et al. Atomic-scale determination of surface facets in gold nanorods. Nature Materials $\mathbf{1 1}$, 930-935 (2012).

[31] Bertolini, J. C. et al. Electronic properties of supported $\mathrm{Pd}$ aggregates in relation with their reactivity for 1,3-butadiene hydrogenation. Catalysis Letters 6, 215-223 (1990).

[32] Piccolo, L., Piednoir, A. \& Bertolini, J.-C. Pd-Au single-crystal surfaces: Segregation properties and catalytic activity in the selective hydrogenation of 1,3-butadiene. Surface Science 592, 169 - 181 (2005).

\section{Acknowledgements}

The authors thank R. Beerthuis and J.W. de Rijk for useful discussion and technical support. N. Masoud is acknowledged for providing the gold catalyst reference data. S. Dussi is thanked for critically reading the manuscript. This project has received funding from the European Research Council (ERC) under the European Union's Horizon 2020 research and innovation programme (ERC-2014-CoG No 648991) and the European Unions Seventh Framework Programme (FP2007-2013)/ERC Advanced Grant Agreement \#291667 HierarSACol. J.v.d.H. also acknowledges the Graduate programme of the Debye Institute for Nanomaterials Science (Utrecht University), which is facilitated by the grant 022.004.016 of the NWO, the Netherlands Organisation for Scientific research. J.J. and F.S. gratefully acknowledge support by the state of Baden-Württemberg through bwHPC (bwunicluster and JUSTUS, RV bw17D011) as well as financial support from the Helmholtz Association.

\section{Author contributions}

JvdH performed the experiments supervised by AvB and PdJ. JJ performed the calculations under the supervision of FS. LO synthesized the nanorods supervised by JvdH. GT assisted in the catalysis experiments. RvD-M performed the ICP-AES measurements. JvdH and PdJ wrote the paper with contributions of all authors.

\section{Competing interests}

The authors declare no competing interests. 